\title{
Tenofovir Treatment Has Lower Risk of Hepatocellular Carcinoma than Entecavir Treatment in Patients with Chronic Hepatitis B: A Systematic Review and Meta-Analysis
}

\author{
Hairong Liu ${ }^{a}$ Yu Shi ${ }^{b}$ John C. Hayden ${ }^{c}$ Paul M. Ryan ${ }^{d} \quad$ Jamal Rahmani ${ }^{e}$ \\ Guangsheng $\mathrm{Yu}^{\mathrm{e}}$ \\ a Department of Oncology, The First Affiliated Hospital of Shandong First Medical \\ University, Jinan, China; ${ }^{b}$ Department of General Surgery, ShanXian Hospital of Traditional \\ Chinese Medicine, Heze City, China; ' $S c h o o l$ of Pharmacy and Biomolecular Sciences, Royal \\ College of Surgeons in Ireland, Dublin, Ireland; ${ }^{\mathrm{d}}$ School of Medicine, University College \\ Cork, Cork, Ireland; e Department of Hepatobiliary Surgery, Shandong Provincial Hospital \\ Affiliated to Shandong First Medical University, Jinan, China
}

\section{Keywords}

Tenofovir · Entecavir · Hepatocellular carcinoma

\begin{abstract}
Purpose: Tenofovir (TDF) and entecavir (ETV) are both equally recommended as first-line treatments for patients with chronic hepatitis B (CHB). They have comparable efficacy in virologic response, but their effect on the development of hepatocellular carcinoma (HCC) in $\mathrm{CHB}$ is controversial. Therefore, we aimed to compare TDF and ETV evaluating the risk of HCC development in $\mathrm{CHB}$ patients. Methods: A systematic literature search was conducted up to November 2019 in MEDLINE/PubMed, SCOPUS, and Web of Science databases without language and time restrictions. DerSimonian and Laird random-effects models were used to estimate combined hazard ratios (HRs) and 95\% Cls. Results: Seven studies containing 35,785 participants were included in this systematic review and meta-analysis. The pooled $\mathrm{HR}(95 \% \mathrm{Cl})$ of HCC in the patients who used TDF versus patients who used ETV was $0.75(0.56-0.96)$. There was no significant heterogeneity detected among the included studies results $\left(I^{2}=47.5 \%\right)$. There was no significant publication bias detected among the included studies (Begg's $p=$ 0.88 and Egger's regression test $p=0.96$ ). Conclusions: Evidence to date suggests that TDF treatment is associated with significantly fewer cases of HCC when compared to ETV.
\end{abstract}




\section{Introduction}

Hepatitis B virus (HBV) infection continues to be a major public health issue worldwide with $>257$ million (or $3.5 \%$ of the world's population) chronically infected with the virus [1]. Cirrhosis, liver failure, or hepatocellular carcinoma (HCC) develops in approximately $15-40 \%$ of infected patients [2]. Others will have lifelong quiescent disease activity not requiring antiviral therapy [3]. However, mortality due to hepatitis is increasing with HBV-related complications accounting for almost 1 million related deaths in 2016 [4]. The recommended indications for treatment are based on 3 parameters: serum HBV DNA concentration, serum ALT concentration, and severity of liver disease [3].

Recommended agents include entecavir (ETV) and tenofovir (TDF), which have higher barriers to resistance than older antivirals such as lamivudine. ETV and TDF are recommended as first-line treatment for chronic hepatitis B (CHB) by practice guidelines [5-7], and both drugs achieve virologic response rates of around 95\%, with very low rates of resistance development alongside good safety profiles [8]. Notwithstanding this clinical equipoise, some important differences in indications exist. ETV should not be used for patients with previous resistance to HBV antivirals where TDF disoproxil fumarate and TDF alafenamide are preferred [3]. TDF disoproxil fumarate is preferred in pregnant women [3]. Conversely, TDF disoproxil fumarate may be less suitable for patients at risk of bone disease or renal impairment. To date, however, there has been no head-to-head randomized control trial comparing the effect of ETV and TDF on long-term clinical outcomes [9].

In the absence of controlled comparisons of these 2 agents, real-world evidence is emerging, albeit inconsistently, of differential rates of development of HCC between ETV-and TDF-based regimens. The very recent publication of several studies in this area [9-12] and the retraction and replacement of a large study [11], with an updated analysis, merit the conduct of an up-to-date review of this research question.

We conduct a systematic review and meta-analysis of empirical studies comparing the effectiveness of ETV monotherapy versus TDF monotherapy in reducing the complication of HCC in patients with CHB infection.

\section{Materials and Methods}

Search Strategy

PubMed/MEDLINE, Scopus, and Web of Science databases were searched up to November 2019 without any language limitations imposed. The literature search contained Mesh and non-Mesh terms (summary provided in online suppl. Table 1; see www.karger.com/doi/10.1159/000507253 for all online suppl. material ). The MOOSE guidelines were followed in the conduct of this meta-analysis [13].

\section{Inclusion Criteria}

Studies that met the following PICOS criteria were included in the meta-analysis: P: CHB patients, I: TDF, C: ETV, Outcome: risk of HCC, and S: observational and randomized controlled trial (RCT) studies. Studies were included in this meta-analysis if they met the following criteria. Review papers, nonhuman studies, editorials, in vitro research, correspondence and case reports, letters without sufficient data, or studies with overlapping data with already included studies were excluded from the meta-analysis. Where multiple reports occurred, the longest follow-up report was included in the meta-analysis.

Data Extraction and Quality Assessment

Two authors independently screened and extracted data from the included studies. Screening was carried out in two steps. First, based on title and abstracts only, and then second, based on a full-text review of potentially eligible studies. Data extraction was performed according to extraction forms. Discrepancies between researchers in screening and data extracting were discussed and resolved with a senior author. The

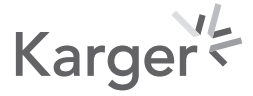




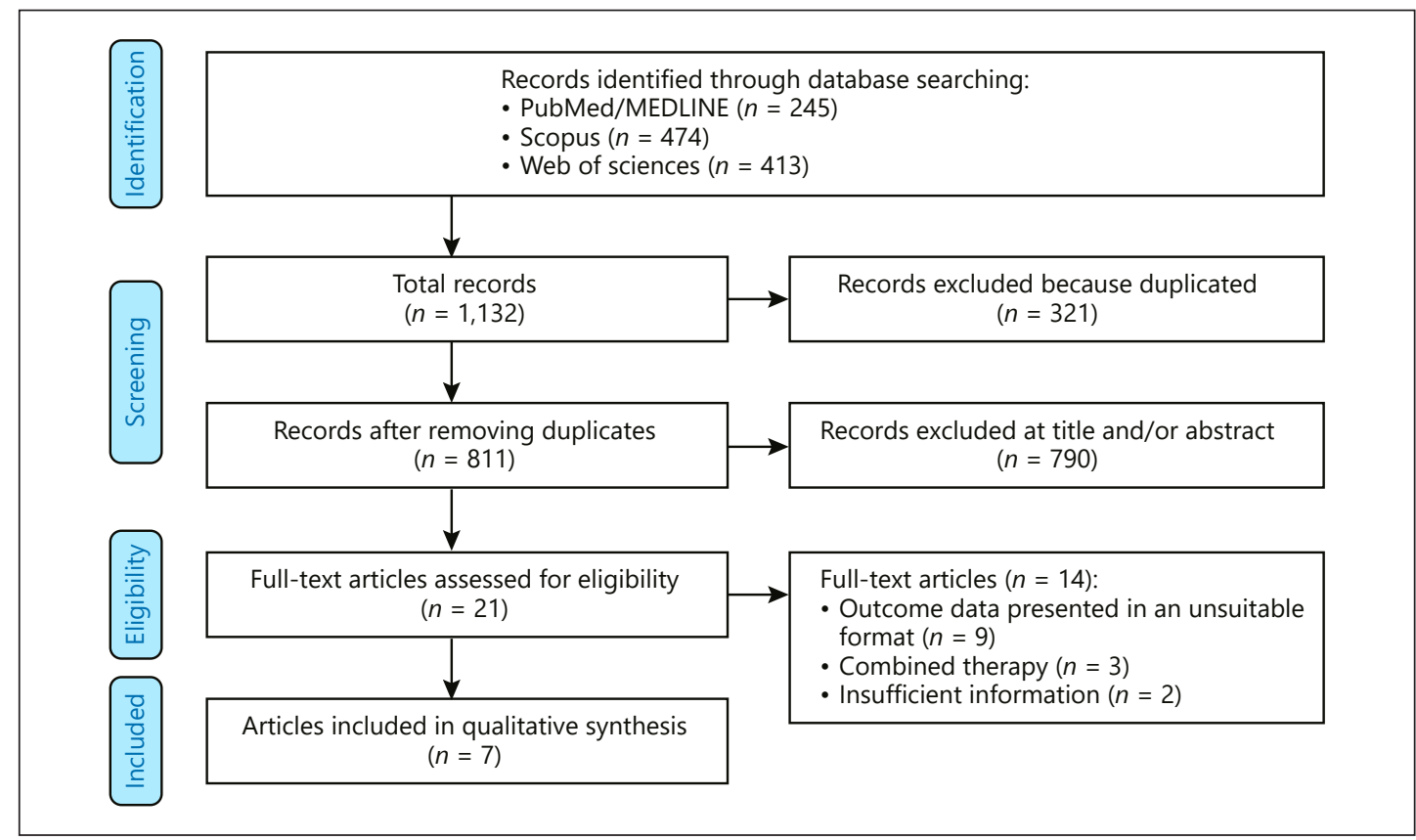

Fig. 1. Flowchart of included studies.

meta-analysis used data from included studies incorporated into fully adjusted models. The NewcastleOttawa Quality Assessment Scale (NOS) was used to assess the quality of the included studies [14].

\section{Statistical Analysis}

The STATA 14.0 statistical software was used in the statistical analysis. We used matched results data for analysis. The results were combined with a random-effects model (DerSimonian and Laird method) [15]. The group that used ETV was considered as the control group. The Cochrane Q test and $I^{2}$ statistic were calculated to estimate heterogeneity among the included studies. The effect of each study on the combined results was evaluated with a sensitivity analysis. Risk of publication bias was evaluated with a Funnel plot, Begg's rank correlation test, and Egger's regression asymmetry test.

\section{Results}

\section{Literature Search}

Figure 1 outlines the flow diagram of studies that were eventually included in the metaanalysis. The comprehensive systematic search results yielded 1,132 primary papers. From these, 321 records were excluded as duplicates. Furthermore, 790 irrelevant records were excluded during the title and/or abstracts screening step. In the full-text screening, 14 studies did not meet our inclusion criteria and were therefore excluded from the meta-analysis, leaving 7 studies containing 35,785 participants were ultimately included in this metaanalysis [10-12, 16-19].

\section{Study Characteristics and Quality Assessment}

Characteristics of the included studies are reported in Table 1. All studies were published between 2017 and 2019. One study was performed in China [16], 4 in Korea [10-12, 17], 1 in Taiwan [18], and 1 was an international, multicentric cohort study [18]. All studies had a retrospective cohort design with a mean length of follow-up of 4 years, with a range from 3.2 


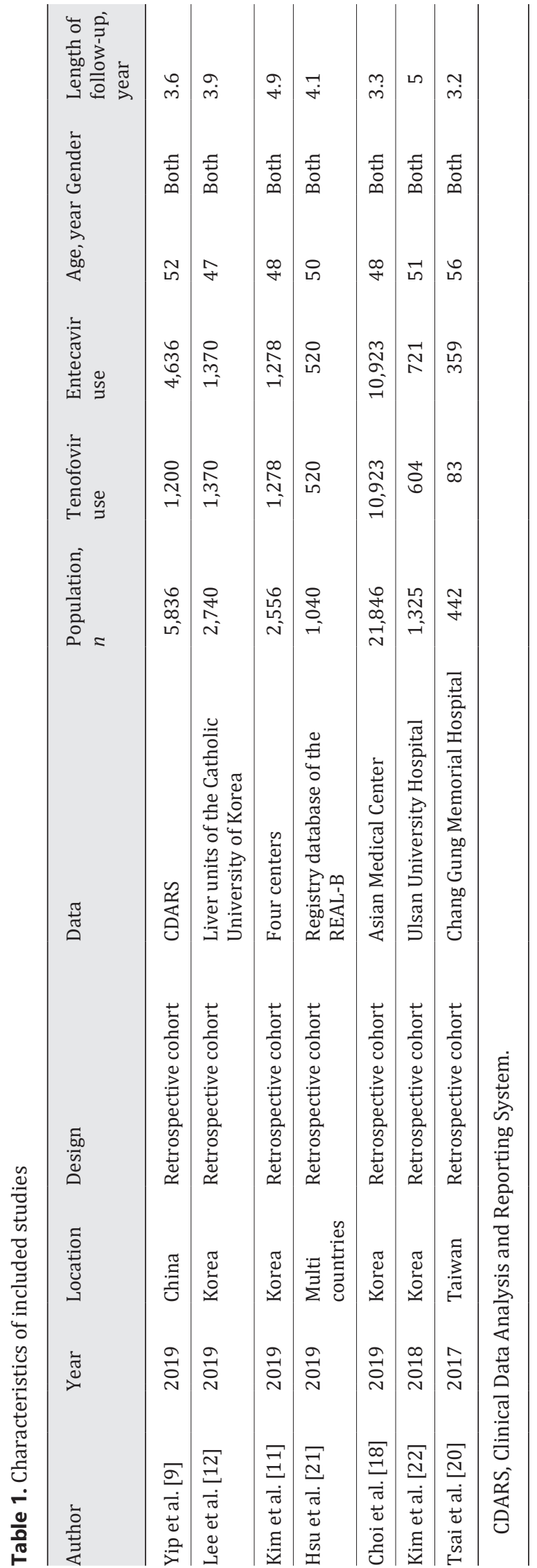




\begin{tabular}{|c|c|c|}
\hline Study, ID & $\mathrm{HR}(95 \% \mathrm{Cl})$ & Weight, \% \\
\hline$\longrightarrow$ & $0.39(0.18,0.84)$ & 8.49 \\
\hline Lee, S. W. (2019) & $1.07(0.51,2.24)$ & 9.04 \\
\hline Kim, S. U. (2019) & $1.02(0.77,1.35)$ & 26.74 \\
\hline Hsu, Y. C. (2019) & $0.89(0.41,1.93)$ & 8.46 \\
\hline Choi, J. (2019) & $0.68(0.60,0.78)$ & 35.54 \\
\hline Kim, B. G. (2018) & $0.53(0.23,1.22)$ & 7.48 \\
\hline Tsai, M. C. (2017) & $0.52(0.16,1.66)$ & 4.26 \\
\hline Overall $\left(R^{2}=47.5 \%, p=0.076\right)$ & $0.75(0.58,0.96)$ & 100.00 \\
\hline NOTE: Weights are from random effects analysis & & \\
\hline 0.163 & 6.13 & \\
\hline
\end{tabular}

Fig. 2. Meta-analysis of the incidence of HCC between the TDF group and ETV groups.

Fig. 3. Funnel plot to assess pub-

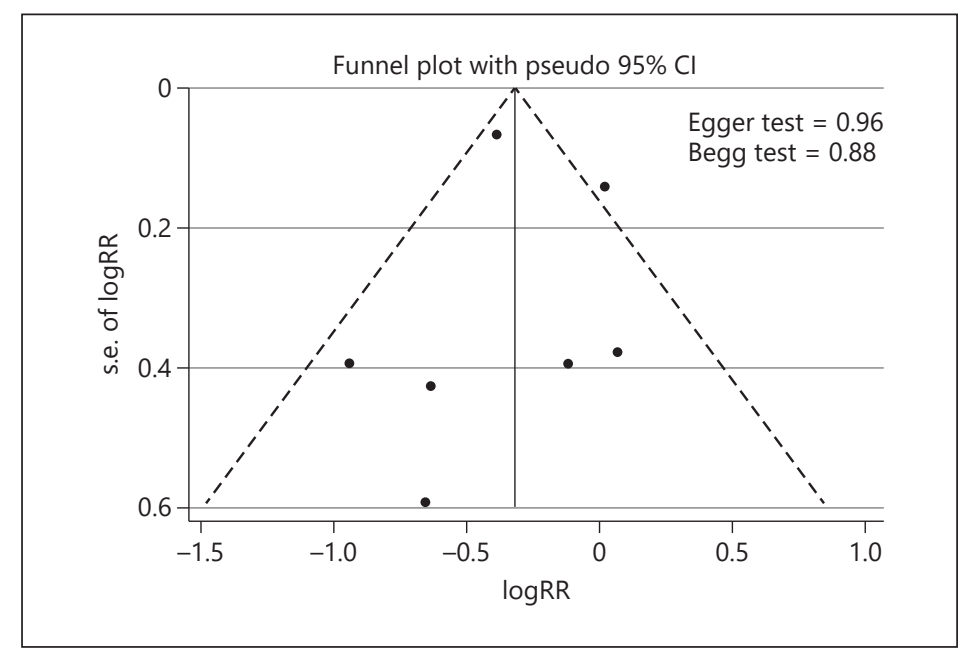
lication bias.

to 5 years. The included studies contained data on 35,785 patients with $\mathrm{CHB}$, of which 15,978 and 19,807 patients used TDF and ETV as nucleos(t)ide analogue therapy, respectively. All studies contained both genders. The mean age of participants was 50.28 years. The quality assessment was performed using NOS, and all studies were found to be of good quality (online suppl. Table 2)

\section{Main Results of the Meta-Analysis}

Seven studies provided data on 35,785 patients with CHB for the meta-analysis [10-12, 16-19]. These studies allowed estimation of hazard ratios (HRs) of HCC development in patients prescribed TDF compared to those prescribed ETV as nucleos $(\mathrm{t})$ ide analogue therapy. The pooled HR ( $95 \% \mathrm{CI})$ of HCC in patients who used TDF versus patients who used ETV was 0.75 (0.56-0.96), in favor of TDF (Fig. 2). Significant heterogeneity was not found among the included studies results $\left(I^{2}=47.5 \%\right)$.

\section{Publication Bias and Sensitivity Analysis}

Funnel plots are shown in Figure 3. There was no significant publication bias detected among the included studies (Begg's $p=0.88$ and Egger's regression test $p=0.96$ ). Finally, 
Liu et al.: TDF, ETV, and HCC

online supplementary Figure 1 shows the results of a sensitivity analysis. No significant differences beyond the limits of $95 \% \mathrm{CI}$ of combined results were found.

\section{Discussion}

The introduction of highly potent antiviral therapeutic regimens has had a substantial effect on the incidence of CHB-driven liver disease, cirrhosis, and HCC. In this context, there is little doubt that TDF and ETV are both effective options in the treatment of CHB, as assessed by intermediate surrogate end points, such as viral load, serology, and hepatic biochemistry. However, whether one is superior to the other in terms of HCC prevention is currently largely unknown. In the absence of head-to-head RCTs, several groups have recently conducted retrospective cohort analyses of $\mathrm{CHB}$ patients receiving these therapies in order to generate real-world data on their comparative effectiveness. These studies have generated varying results and are not unified in their conclusions. In line with this, the present systematic review and meta-analysis set out to identify and synthesize all available retrospective cohort data that concerned the efficacy of these 2 high-potency antivirals in the context of patients with CHB. Following the identification of 7 similarly designed studies containing 45,274 participants, our meta-analysis found that TDF use was associated with a lower incidence of HCC when compared to ETV (HR 0.75, 95\% CI 0.58-0.96).

Although TDF-treated cohorts were found to develop fewer cases of HCC in the combined analysis model, just 2 of the 7 included studies demonstrated the same significant effect independently $[9,17]$, while the remaining studies identified no such difference between the 2 therapeutic options. An editorial by Flemming et al. [20] suggested that many previous retrospective cohort studies did not contain the number of event rates that were required in order to detect a superiority between the therapeutic options. Indeed, Yip et al. [9] examined by far the largest population of antiviral-treated individuals and also reported the most substantial efficacy imbalance of all the identified studies, with HCC incidences of 0.6 and $4.9 \%$ for TDF and ETV, respectively. However, these results may have been influenced by the unbalanced nature of the 2 groups, with ETV-treated patients representing $95 \%$ of the total examined cohort. As a result, there were just 8 recorded cases of HCC within the TDF-treated cohort, as compared to 1,386 in the ETV group. Therefore, it appears likely that the HR reported may change as data from more TDF-treated patients and, in turn, HCC cases are included.

In line with the current meta-analysis, the results of the retrospective analysis of administrative data by Choi et al. [17] suggest that TDF prescription resulted in a third fewer cases of HCC when compared to ETV. In general, ETV performed comparably in propensity scoreadjusted models between this study and that reported by Lee et al. [12] (0.05\% probability of HCC at 4 and 5 years, respectively) [11]; however, TDF began to plateau and diverge from ETV at year 2 of intervention in the former study, ultimately plateauing at around $0.03 \%$. The source of this significant disparity is not entirely clear at present; however, Lee et al. [12] indicate that the criterion to initiate treatment in their cohort may have been more stringent than others, which may mean that earlier initiation of TDF is required in order to see the benefits against its comparator. Nevertheless, Choi et al. [17] went on to further validate their result in a hospital-based cohort that was well characterized both in terms of disease severity and viral load status, an exercise that adds considerable robustness to the conclusions drawn.

Previous meta-analyses have evaluated differential HCC incidence with ETV and TDF treatment [21-24]. Although their findings were broadly consistent with our findings, a strength of our study is the inclusion of the large cohort study that has recently been published [9]. This has not featured in other reviews. Our study also has the advantage of not using unadjusted crude data to estimate risks. We also used propensity score matching to minimize 
the risk of selection bias in our analysis. The studies included and the meta-analysis itself have several notable strengths and limitations associated with them. In general, the included studies all reported a similar design, with roughly comparable duration of follow-up, cohort sizes, and cohort compositions. In addition, substantial efforts have been made to control for potentially confounding variables in each of the studies, adjusting for age (as ETV may have been preferentially selected for older patients due to potential adverse bone effects associated with TDF), disease severity/propensity score, and statin or metformin use, along with many other potentially important factors. However, due to the nature of these observational studies, there may be additional unidentified and unmeasured factors that have impacted substantially upon the outcome. Our findings must be interpreted within the context that all included studies are based on observational cohort studies, themselves at risk of selection bias. Indeed, Yip et al. [9] acknowledge in their discussion that a diagnosis of cirrhosis was not readily available for the vast majority of patients, as the confirmatory techniques are not commonplace in clinical practice in the assessed region. This is indeed a shortcoming of these retrospective studies and an aspect that could be remedied effectively through an RCT. The mean reported follow-up times ranged between 3 and 5 years, and, although this appears to have been sufficiently long to incur an analyzable number of HCC cases, it will be important that subsequent studies aim to understand the longer term effects of both treatments. In addition, the studies included in this meta-analysis are composed almost exclusively of Asian cohorts, and, although several Asian countries commonly report concerningly high rates of $\mathrm{CHB}$, this limits the generalizability of the result and indicates that future work is required in regions with similarly high incidence rates, such as Africa. Similarly, it will now be important to tease out if each genotype of the HBV responds in a similar fashion. Finally, the majority of studies included in the present review examined cohorts that were composed entirely of treatment-naïve patients. This is important since it is possible that patients may require a therapy switch from one to another due to intolerable side effects or development of viral resistance, both of which may have significant impact upon the efficacy of the therapy and, therefore, the risk of HCC development.

\section{Conclusion}

TDF and ETV have both proven to be effective therapeutic options in the management of CHB, as assessed by viral load and hepatic biochemistry parameters. However, there is little understanding of whether one of these antivirals is superior to the other in terms of HCC prevention. The current systematic review and meta-analysis aimed to identify and synthesize all available retrospective cohort data that assessed risk of HCC diagnosis in CHB patients receiving either TDF or ETV. We uncovered 7 suitable studies that included data from 45,274 CHB patients and conducted a meta-analysis that combined the reported HRs. This demonstrated that TDF treatment was associated with significantly less cases of HCC when compared to those treated with ETV (HR 0.76, 95\% CI 0.56-0.96), suggesting that the former may be the more effective choice in such patient cohorts. However, well-designed and large-scale (due to inherently low event rates) RCTs are now warranted to further validate this result by attempting to exclude any remaining potential bias or confounding.

\section{Acknowledgment}

There are no acknowledgments to declare.

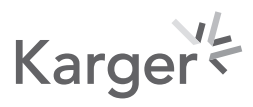




\begin{tabular}{|c|c|}
\hline \multicolumn{2}{|l|}{ Liver Cancer 2020;9:468-476 } \\
\hline DOI: 10.1159/000507253 & $\begin{array}{l}\text { (c) } 2020 \text { The Author(s). Published by S. Karger AG, Basel } \\
\text { www.karger.com/lic }\end{array}$ \\
\hline
\end{tabular}

\section{Liver Cancer}

Liu et al.: TDF, ETV, and HCC

\section{Statement of Ethics}

The authors have no ethical conflicts to disclose. It is systematic review.

\section{Disclosure Statement}

The authors declare no conflict of interest.

\section{Funding Sources}

The authors received grants from the Shandong Provincial Natural Science Foundation [grant numbers ZR2014HQ019].

\section{Author Contributions}

J.R. and G.Y. idea and analysis. H.L. and Y.S. data extraction and searches. J.C.H. and P.M.R. writing. All authors reviewed the final draft.

\section{References}

1 Organization WH. Global hepatitis report 2017. World Health Organization; 2017.

2 Lok AS. Chronic hepatitis B. N Engl J Med. 2002 May;346(22):1682-3.

3 Seto WK, Lo YR, Pawlotsky JM, Yuen MF. Chronic hepatitis B virus infection. Lancet. 2018 Nov;392(10161): 2313-24.

4 World Health Organization. Global Health Estimates 2016: Deaths by Cause, Age, Sex, by Country and by Region, 2000-2016. Geneva: 2018. 2019.

5 Terrault NA, Lok AS, McMahon BJ, Chang KM, Hwang JP, Jonas MM, et al. Update on prevention, diagnosis, and treatment of chronic hepatitis B: AASLD 2018 hepatitis B guidance. Hepatology. 2018 Apr;67(4):1560-99.

6 Sarin SK, Kumar M, Lau GK, Abbas Z, Chan HL, Chen CJ, et al. Asian-Pacific clinical practice guidelines on the management of hepatitis B: a 2015 update. Hepatol Int. 2016 Jan;10(1):1-98.

7 Liver EAEuropean Association for the Study of the Liver. Electronic address: easloffice@easloffice.eu; European Association for the Study of the Liver. EASL 2017 Clinical Practice Guidelines on the management of hepatitis B virus infection. J Hepatol. 2017 Aug;67(2):370-98.

8 Pol S, Lampertico P. First-line treatment of chronic hepatitis B with entecavir or tenofovir in 'real-life' settings: from clinical trials to clinical practice. J Viral Hepat. 2012 Jun;19(6):377-86.

9 Yip TC, Wong VW, Chan HL, Tse YK, Lui GC, Wong GL. Tenofovir is associated with lower risk of hepatocellular carcinoma than entecavir in patients with chronic HBV infection in China. Gastroenterology. 2020 Jan;158(1): 215-25.e6.

10 Kim BG, Park NH, Lee SB, Lee H, Lee BU, Park JH, et al. Mortality, liver transplantation and hepatic complications in patients with treatment-naïve chronic hepatitis B treated with entecavir vs tenofovir. J Viral Hepat. 2018 Dec;25(12):1565-75.

11 Kim SU, Seo YS, Lee HA, Kim MN, Lee YR, Lee HW, et al. A multi-center study of entecavir vs. tenofovir on prognosis of treatment-naïve chronic hepatitis B in South Korea. J Hepatol. 2019 Sep;71(3):456-64.

12 Lee SW, Kwon JH, Lee HL, Yoo SH, Nam HC, Sung PS, et al. Comparison of tenofovir and entecavir on the risk of hepatocellular carcinoma and mortality in treatment-naïve patients with chronic hepatitis B in Korea: a large-scale, propensity score analysis. Gut. 2019 Oct;gutjnl-2019-318947.

13 Stroup DF, Berlin JA, Morton SC, Olkin I, Williamson GD, Rennie D, et al. Meta-analysis of observational studies in epidemiology: a proposal for reporting. Meta-analysis Of Observational Studies in Epidemiology (MOOSE) group. JAMA. 2000 Apr;283(15):2008-12.

14 Stang A. Critical evaluation of the Newcastle-Ottawa scale for the assessment of the quality of nonrandomized studies in meta-analyses. Eur J Epidemiol. 2010 Sep;25(9):603-5.

15 Jackson D, White IR, Thompson SG. Extending DerSimonian and Laird's methodology to perform multivariate random effects meta-analyses. Stat Med. 2010 May;29(12):1282-97.

16 Yip TCF, Wong VWS, Tse YK, Chan H, Wong GLH. Tenofovir treatment has lower risk of hepatocellular carcinoma than entecavir treatment in patients with chronic hepatitis B. J Hepatol. 2019;70(1):e128.

17 Choi J, Kim HJ, Lee J, Cho S, Ko MJ, Lim YS. Risk of Hepatocellular Carcinoma in Patients Treated With Entecavir vs Tenofovir for Chronic Hepatitis B: A Korean Nationwide Cohort Study. JAMA Oncol. 2019 Jan;5(1):30-6.

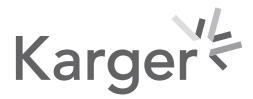


18 Tsai MC, Chen CH, Hu TH, Lu SN, Lee CM, Wang JH, et al. Long-term outcomes of hepatitis B virus-related cirrhosis treated with nucleos(t)ide analogs. J Formos Med Assoc. 2017 Jul;116(7):512-21.

19 Hsu YC, Wong GL, Chen CH, Peng CY, Yeh ML, Cheung KS, et al. Tenofovir Versus Entecavir for Hepatocellular Carcinoma Prevention in an International Consortium of Chronic Hepatitis B. Am J Gastroenterol. 2020 Feb; 115(2):271-80.

20 Flemming JA, Terrault NA. Tenofovir vs Entecavir for Hepatocellular Carcinoma Prevention in Patients With Chronic Hepatitis B: One of These Things Is Not Like the Other. JAMA Oncol. 2019 Jan;5(1):17-8.

21 Li M, Lv T, Wu S, Wei W, Wu X, Ou X, et al. Tenofovir versus entecavir in lowering the risk of hepatocellular carcinoma development in patients with chronic hepatitis B: a critical systematic review and meta-analysis. Hepatol Int. 2020 Jan;14(1):105-14.

22 Zhang Z, Zhou Y, Yang J, Hu K, Huang Y. The effectiveness of TDF versus ETV on incidence of HCC in CHB patients: a meta analysis. BMC Cancer. 2019 May;19(1):511.

23 Chen MB, Wang H, Zheng QH, Zheng XW, Fan JN, Ding YL, et al. Comparative efficacy of tenofovir and entecavir in nucleos(t)ide analogue-naive chronic hepatitis B: A systematic review and meta-analysis. PLoS One. 2019 Nov;14(11):e0224773.

24 Wang X, Liu X, Dang Z, Yu L, Jiang Y, Wang X, et al. Nucleos(t)ide analogues for reducing hepatocellular carcinoma in chronic hepatitis b patients: a systematic review and meta-analysis. Gut Liver. 2020 Mar;14(2): 232-47. 